Rabaska

Revue d'ethnologie de l'Amérique française

\title{
Hommage d'un comédien à son grand-oncle, le peintre-illustrateur québécois Edmond-J. Massicotte (1875-1929)
}

\section{Yves Massicotte}

Volume 4, 2006

URI : https://id.erudit.org/iderudit/201770ar

DOI : https://doi.org/10.7202/201770ar

Aller au sommaire du numéro

Éditeur(s)

Société québécoise d'ethnologie

ISSN

1703-7433 (imprimé)

1916-7350 (numérique)

Découvrir la revue

Citer cette note

Massicotte, Y. (2006). Hommage d'un comédien à son grand-oncle, le peintre-illustrateur québécois Edmond-J. Massicotte (1875-1929). Rabaska, 4, 123-130. https://doi.org/10.7202/201770ar d'utilisation que vous pouvez consulter en ligne. 
Points de vue / exposition

\section{Edmond-Joseph Massicotte, illustrateur (1875-1929) Une exposition, un livre, deux points de vue}

L'exposition Edmond-Joseph Massicotte, illustrateur, qui a récemment été présentée au Musée national des beaux-arts du Québec, a profondément ému le comédien Yves Massicotte, qui a bien voulu faire part aux lecteurs de RABASKA de son témoignage sur son illustre parent, et qui, en professionnel accompli, sait regarder une œuvre, la situer dans son contexte et l'apprécier pour ce qu'elle est plutôt que ce que le goût du jour veut y voir. Nous avons aussi demandé à Bernard Genest, ethnologue réputé qui a spécialement étudié les dessins de l'artiste, dont il a notamment publié les résultats dans son livre Massicotte et son temps, de nous livrer son point de vue d'expert sur cette exposition et sur le livre qui l'accompagne.

\section{Hommage d'un comédien à son grand-oncle, le peintre-illustrateur québécois Edmond-J. Massicotte (1875-1929)}

Yves MASSICOTTE

Montréal

L'exposition des œuvres majeures d'Edmond-J. Massicotte au Musée national des beaux-arts du Québec, en 2005 et 2006, fut à mes yeux une résurgence artistique d'une très grande importance. Mes visites à cette exposition m'ont profondément secoué, et aujourd'hui, au moment d'écrire cet hommage, mon émotion ne s'est pas encore résorbée ; au contraire, elle suscite en moi des débats d'idées et un brassage de mes valeurs artistiques que je tente quotidiennement de démêler et d'ordonner.

L'hommage que je veux rendre à ce grand-oncle, que je n'ai malheureusement pas connu de son vivant, débutera - et j'y tiens - par un coup de chapeau à son petit-fils Jean-François Pothier, ébéniste, qui par sa ténacité, 
son opiniâtreté devrais-je dire, fut l'inspirateur de cet heureux événement artistique. Sans lui, l'exposition Massicotte à Québec n'aurait probablement pas eu lieu. C'est Jean-François qui a talonné et su convaincre les responsables du Musée national des beaux-arts du Québec d'oser remettre l'artiste EdmondJ. Massicotte « à l'affiche », ainsi qu'on le dit dans mon métier. Quel exemple et quel encouragement pour tous ceux et celles qui, comme lui, souffrent d'un sérieux handicap physique !

C'est lors d'un souper familial, en janvier 2005, chez mon frère aîné, que notre cousin Jean-François nous annonça avec joie et enthousiasme qu'une grande exposition des œuvres d'Edmond-J. aurait lieu au cours de l'année à Québec, et qu'en vue de sa réalisation il était en étroite collaboration avec un des conservateurs du musée. Nous, ses cousins et cousines Massicotte, en fûmes tous fort réjouis.

Cependant, je dois l'avouer, $\mathrm{j}$ 'étais en mon for intérieur un peu sceptique quant à la réussite possible de son projet. Quelques années auparavant, JeanFrançois avait tenté d'organiser dans le vieux Montréal une exposition d'Edmond-J. Massicotte qui n'avait pas eu grand succès. Sans doute lui manquait-il les œuvres majeures de l'artiste, lesquelles appartiennent toutes au Musée national des beaux-arts du Québec, et qu'à cette exposition de Montréal il y avait beaucoup trop de reproductions et pas assez d'œuvres originales. Peut-être aussi que l'heure de "ressusciter » la mémoire de Massicotte n'avait pas encore sonné... D'ailleurs, comment voulez-vous qu'un peuple constamment tourmenté et « enfirouâpé » par tout ce qui sort de bon et de mauvais de la télé et des autres médias, et par les multiples sollicitations, tant commerciales que politiques qui nous assaillent de tous côtés, puisse avoir le goût et la sérénité nécessaires pour apprécier les œuvres pacifiques d'un illustrateur de son passé, si grand soit-il, et dont on s'est tant moqué à une certaine époque à cause, disait-on, de « ses imageries passéistes toutes imbibées d'une sainte onction religieuse ».

C'est donc avec réticence et un peu craintivement que je me suis rendu au Musée national des beaux-arts du Québec - car, malgré tout, j'y tenais trois jours après le vernissage où, paraît-il, il y avait eu grande foule. Et parmi cette foule, des personnalités bien connues de Monsieur-tout-le-monde. Le vent commencerait-il à tourner ? L'heure serait-elle venue d'apprécier à sa juste valeur un artiste québécois méconnu de la présente génération ? Peutêtre. Mais dans la vie, mes amis, il ne faut jurer de rien...

En ouvrant la porte d'entrée de la salle d'exposition, la première chose qui me sauta aux yeux fut la qualité de la présentation. La belle ordonnance des tableaux. C'était évident, des gens d'expérience et de goût avaient travaillé à la mise en place des œuvres exposées. À partir de celles qui étaient mineures 


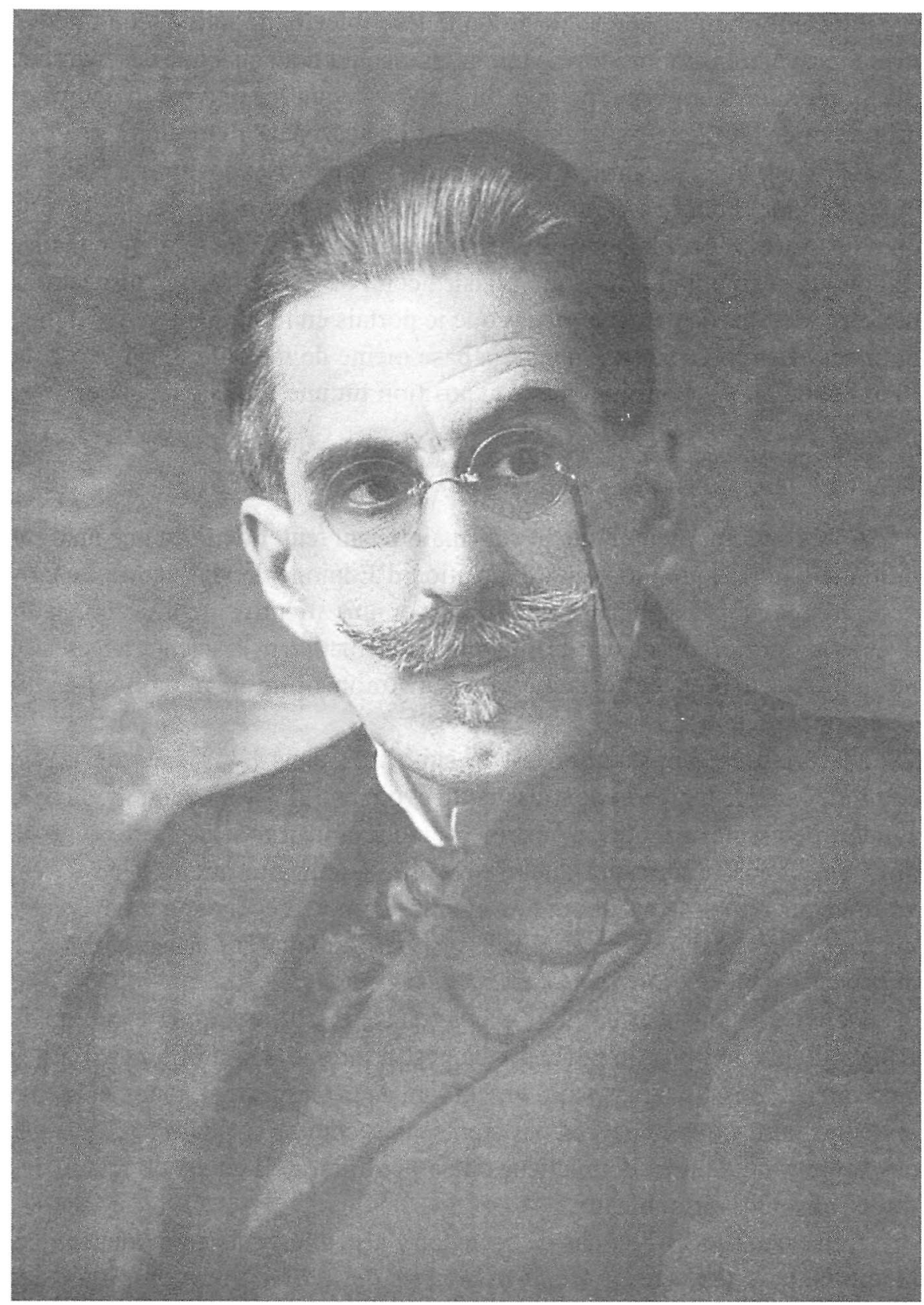

Edmond-Joseph Massicotte à l'époque de sa maturité. Document imprimé gracieusement fourni par Yves Massicotte. 
et plutôt informatives, on avait ordonné les tableaux de telle façon que les visiteurs avaient l'impression de gravir un escalier dont chacune des marches offrait un intérêt nouveau, pour enfin arriver devant les œuvres majeures et originales de l'artiste. Évidemment, gradation aussi dans l'émotion ressentie.

Le plaisir que j'ai éprouvé en parcourant la salle d'exposition n'était pas qu'esthétique, comme ce fut le cas à l'exposition des gravures de Rubens dont les sujets n'éveillaient rien en moi de personnel. Cette fois, mon plaisir esthétique se doublait d'un autre plaisir : celui de voir et reconnaître sur les tableaux de Massicotte des images que je portais en moi, que j'avais un peu oubliées et qui pourtant formaient la base même de ma culture. Si le but de l'Art est de plaire, pour moi, cette exposition fut une franche réussite.

Après ce long préambule, je vais maintenant tenter de faire une analyse rationnelle de ce que j'ai vu à l'exposition d'Edmond-J. Massicotte. Je vais m'arrêter devant ses tableaux et les observer plus attentivement. J'ai bien dit « tenter », car $j$ 'ai conscience de n'avoir ni la formation ni la science nécessaire pour mener à bien cette tâche : ce sera mon opinion personnelle qui s'exprimera ici.

Ma première constatation est la suivante : dans les scènes de la vie courante que les tableaux de Massicotte évoquent, il n'y a aucune violence, aucune personne malade ou infirme, aucune allusion à la guerre ou à la politique, aucune chicane, aucune révolte, aucun nu, aucun vice. C'est vraiment un temps de paix pour tout et tous. La mort, cependant, est évoquée, mais avec beaucoup de pudeur. Elle inspira d'ailleurs à l'artiste un de ses plus beaux tableaux : le Saint Viatique à la campagne.

Deuxième constatation : il n'y a pas dans les tableaux que j'examine de scènes de pêche ; pas de pêcheurs dans son monde. On a affaire ici à des cultivateurs, des « terriens » qui vivent loin de la Gaspésie. La mer, et même le fleuve Saint-Laurent ne sont pas représentés, comme si, peut-être, c'étaient des sources de danger, de malheur ou de perdition... Il en est de même des forêts, absentes des tableaux.

Autre chose : il n'y a pas de scène où l'argent est mis en évidence, pas même dans le tableau Le Magasin général de jadis où on voit plutôt des joueurs de dames que des spéculateurs. Il n'y a pas de banques ou de banquiers, pas de Séraphin Poudrier, pas de quêteux, pas même l'ombre d'un bas de laine!

On ne voit dans les tableaux de Massicotte que des gens heureux, en bonne santé, paraissant ignorer tous les dangers et les épreuves de la vie humaine. Ce sont de simples paysans, ni riches ni pauvres, vivant leurs petits 
moments de bonheur avec simplicité et docilité, comptant sans doute, comme des enfants, sur leurs pasteurs pour les protéger et veiller sur eux avec bienveillance et attention. Un climat de joie imprègne chaque tableau : on y rit, on y chante, on y danse, on y prie, on y banquette, on s'aime, on se marie. Tout le contraire du monde des puritains !

Après ces quelques observations on ne peut s'empêcher de se poser des questions. Celles-ci par exemple : où, diantre, ces scènes peuvent-elles bien se situer? Pas en Gaspésie en tout cas, ni dans Charlevoix, il n'y a aucune montagne dans les tableaux. Mais, comme notre pays est vaste et diversifié, cherchons ailleurs... Peut-être alors dans une de nos vieilles paroisses sises le long du fleuve, mais loin derrière, dans les rangs et dans la plaine... Aux alentours de Saint-Stanislas, comté de Champlain, par exemple... (l'artiste avait de la parenté dans cette région-là, mais chut !... c'est un tuyau qui me vient de ma mère).

Bon. Disons qu'il s'agit de ce coin-là. Très bien. Mais à quelle époque l'artiste a-t-il bien pu situer ses scènes ? J'oserai dire : à aucune exactement. Il les a situées dans un " ancien temps », imprécis et surtout mythique. Entre 1860 et 1920 à peu près. À mon avis, l'artiste Massicotte s'est construit de son vivant - avec tout ce qu'il observait autour de lui de réel et d'imaginaire, avec aussi les renseignements précieux que lui fournissait son frère É.-Z. [Édouard-Zotique Massicotte], archiviste émérite, sur les mœurs, coutumes, vêtements, traditions de «l'ancien temps »-il s'est construit, dis-je, un refuge sacré, un monde idyllique, un havre de paix où ses valeurs personnelles, humaines et religieuses pouvaient, en toute sécurité s'épanouir et s'exprimer librement ; un lieu idéal de bonheur et de beauté où n'entreraient jamais les laideurs de la réalité dans lesquelles patauge le monde des « Bougons ». Un refuge. Je tiens à mon idée.

Tous les grands artistes, qu'ils soient écrivains, musiciens, peintres, sculpteurs, éprouvent le même impérieux besoin : fuir ! Fuir à tout prix, par leur art, une réalité décevante; tous recherchent passionnément ce qui est bon, beau et pur, pour parler comme Mozart. Et cette fuite, c'est, en fait, le désir de retrouver, dans un ailleurs rêvé, un paradis perdu, un âge d'or, et, dans ce cas-ci... le Bon Vieux Temps tel que le rêvaient nos grands-parents.

Les œuvres de Massicotte, même si elles semblent très concrètes, ne sont cependant pas des œuvres réalistes. Le style l'est peut-être, mais pas l'esprit des œuvres. Dans toutes, il y a transposition. Elles sont idéalistes et même surréalistes, en ce sens que très peu de choses n'ont vraiment existé telles qu'il les représentaient. Bien sûr, ces scènes, nous les avons tous plus ou moins confusément en nous (je parle surtout pour les gens de ma génération, car, pour les autres, plus jeunes ou de races différentes, ces œuvres ne représentent qu'un intérêt esthétique, ou même qu'une curiosité amusante), 
mais pour ceux et celles que cela concerne, le mérite de l'artiste est d'avoir su ranimer en nous ces scènes afin que nous puissions le suivre dans son rêve, celui de retrouver avec lui une époque et un lieu où le bonheur, la beauté et la paix auraient existé vraiment. En effet, ces scènes, ou ces images qui sont en nous, cueillies dans la vraie vie, ressemblent aux évocations artistiques de Massicotte. Comme lui, nous avons ressenti d'agréables émotions en visitant des cabanes à sucre, en revoyant au cinéma des veillées d'autrefois, en revivant des retours de messes de minuit, des réveillons de Noël, et, les plus chanceux, en causant le long d'une route à la campagne avec une jolie dame faisant sa fournée comme dans le Bon Vieux Temps ! Je connais même quelqu'un qui se souvient d'avoir vu des cultivateurs dans leurs champs arrêter leurs travaux quand la cloche du village sonnait midi, pour réciter l'Angélus.

Voir ou ressentir des choses si belles soient-elles, tout le monde peut le faire, mais avoir le talent de les transposer sur un tableau, dans une œuvre d'art, c'est, comme on dit communément, une autre paire de manches ! Il faut l'inspiration, la technique, le savoir-faire, et ça, Massicotte en avait beaucoup. Je reparlerai de son savoir-faire un peu plus loin...

Quoi qu'on ait dit sur cet artiste, une chose est certaine, il a inspiré beaucoup de gens : des cinéastes, des réalisateurs de séries télévisées, des sculpteurs, des illustrateurs, des dessinateurs de costumes, des décorateurs, qui l'ont sans vergogne copié et imité. Et n'avouant jamais la source de leurs réalisations. Dans d'autres mondes, on crierait au piratage ! Je crois cependant qu'être ainsi imité est une preuve indirecte de son grand talent.

Pauvre toi ! Il était assez riche pour se faire voler !... me dirait sûrement le poète Félix Leclerc. Et l'écho répondrait : ... Assez fort aussi pour résister aux pires moqueries!

Prenons un peu le temps maintenant de nous pencher sur sa technique artistique, sur son savoir-faire - j'y faisais allusion il y a un instant -, car sa technique me paraît essentielle pour bien apprécier l'art de Massicotte.

Edmond-J. travaillait principalement au crayon et à la plume, et utilisait le lavis pour ses ciels, ses jeux d'ombres et de clartés, ses dégradés, et pour tous les détails que l'on remarque moins, soit sur les tapis, les planchers, les vêtements, et sur les murs. Le blanc, le gris et le noir dominent, avec toutes les nuances que cela apporte.

J'ai souvent entendu des gens se demander : «Pourquoi n'a-t-il pas mis de la couleur sur ses tableaux ? Pourquoi avoir utilisé uniquement le noir et le blanc ? " Je répondrai par une question : pourquoi de grands cinéastes 
ont-ils préféré tourner leurs films en noir et blanc plutôt qu'en couleur? Pourquoi une photographie artistique nous accroche-t-elle davantage quand elle est en noir et blanc? C'est la preuve que ce n'est pas une pauvreté d'inspiration, mais un choix délibéré que l'artiste a voulu. D'autres peintres trouvent peut-être dans la couleur et l'abstraction leur " porte d'évasion ", respectons-les, mais admirons aussi ceux qui choisissent une autre voie celle du noir et blanc - aussi lumineuse quand ils atteignent leur but.

Puisque j'ai fait allusion au cinéma, je continuerai dans cette veine. Dans chacun des tableaux de Massicotte, on voit qu'il y a une mise en scène très soignée. C'est comme si on arrêtait brusquement le film sur une image en particulier. En imagination, on peut voir les personnages évoluer, entrer et sortir avant et après l'arrêt du film. C'est fascinant. On entend même fuser les rires, les cris, les chansons, les musiques, et, plus discrètement, le bruit des baisers, des prières, la sonnette précédant le Saint Viatique, et aussi les chants des oiseaux, le caquetage des poules, le cri des grillons dans les champs.

Massicotte, tout en étant un excellent illustrateur sachant évoquer et représenter les choses, était un remarquable metteur en scène : la mise en place de chaque tableau est parfaite. Elle est composée avec goût. Elle est dépouillée et bien ordonnée. Le centre d'intérêt est toujours en évidence. Tous les personnages sont en situation. Massicotte n'avait pas peur des grosses distributions, il y a beaucoup de monde dans ses tableaux. Mais il n'y a pas de remplissage inutile. Tous les personnages sont vivants, chacun bien à sa place, fraternellement unis, dominant allègrement l'hiver québécois par des jeux, des veillées, des banquets, des prières, des messes. Massicotte n'exprime pas la solitude de l'être humain, mais la joie de vivre d'un petit peuple, uni et solidaire dans sa lutte pour sa survie.

Et cet artiste, sans conteste, savait dessiner. Le trait de son dessin est ferme, net. Les corps sont bien proportionnés, les perspectives toujours respectées, les « décors » juste assez évoqués pour remplir leur rôle de décors. Bref, n'ayons pas peur des mots, c'est un art inspiré de l'art classique que pratiquait Massicotte. Les sujets de ses tableaux n'ont certes pas la grandeur ni la magnificence de ceux d'un Nicolas Poussin, ou d'un Louis David, ses personnages sont plus humbles, plus modestes, plus plébéiens. Mais la composition des scènes et la qualité du dessin sont nettement influencées par ces grands artistes. Si le dessin est l'art des grands maîtres, comme je l'ai lu quelque part, Edmond-J. Massicotte, à sa manière, faisait partie de ce club sélect.

Je terminerai en disant qu'il est un des gardiens de notre mémoire collective.

Paix à son âme. 


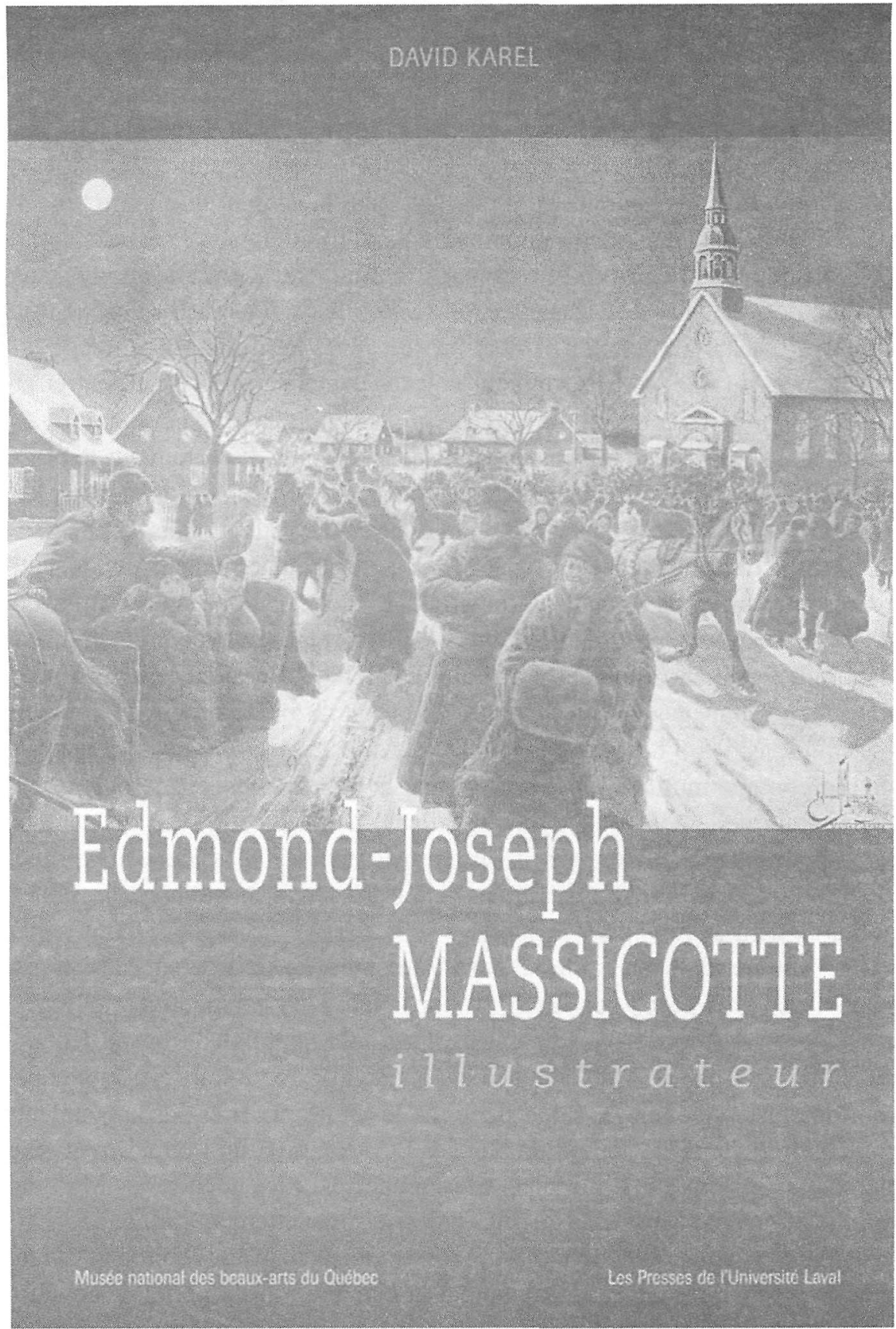

Première de couverture de l'ouvrage publié à l'occasion de l'exposition Edmond-Joseph Massicotte, illustrateur, présentée au Musée national des beaux-arts du Québec.

Illustration : Le Retour de la messe de Minuit, 1919. 